\title{
Data-Driven Additive Manufacturing Constraints for Topology Optimization
}

\author{
Benjamin M Weiss \\ University of Washington \\ Seattle, WA, USA \\ Mark A Ganter \\ University of Washington \\ Seattle, WA, USA
}

\author{
Joshua M Hamel \\ Seattle University \\ Seattle, WA, USA
}

\author{
Duane W Storti \\ University of Washington \\ Seattle, WA, USA
}

\begin{abstract}
The topology optimization (TO) of structures to be produced using additive manufacturing (AM) is explored using a data-driven constraint function on the minimum producible size of small features in different shapes and orientations. This shape- and orientation-dependent manufacturing constraint, derived from experimental data, is implemented within a TO framework using a modified version of the Moving Morphable Components (MMC) approach. Because the analytic constraint function is fully differentiable, gradient-based optimization can be used. The MMC approach is extended in this work to include a "bootstrapping" step, which provides initial component layouts to the MMC algorithm based on intermediate Solid Isotropic Material with Penalization (SIMP) topology optimization results. This "bootstrapping" approach improves convergence compared to reference MMC implementations. Results from two compliance design optimization example problems demonstrate the successful integration of the manufacturability constraint in the MMC approach, and the optimal designs produced show minor changes in topology and shape compared to designs produced using fixed-radius filters in the traditional SIMP approach. The use of this data-driven manufacturability constraint makes it possible to take better advantage of the achievable complexity in additive manufacturing processes, while resulting in typical penalties to the design objective function of around only $2 \%$ when compared to the unconstrained case.
\end{abstract}

\begin{tabular}{cl}
$\begin{array}{l}\text { NOMENCLATURE } \\
\mathbf{c}^{k}\end{array}$ & Parameters for the $k$ th morphable component \\
$E ; E_{\min }$ & Elastic modulus of fully dense; void material \\
$\mathbf{F}$ & Applied load \\
$f$ & Objective function (compliance) \\
$\bar{f}$ & Thresholded compliance \\
$H$ & Smoothed Heaviside function \\
$\mathbf{K} ; \mathbf{k}_{e}$ & Global stiffness matrix; stiffness matrix for element $e$ \\
$\mathbf{k}_{0}$ & Stiffness matrix for a fully-dense element \\
$l$ & Feature/component length \\
$l_{\min }$ & Minimum allowed initial component length \\
$p$ & Density penalization exponent \\
\multicolumn{1}{c}{$S(l, \theta)$} & Data-driven continuous constraint function
\end{tabular}




$\begin{array}{ll}t ; t^{k} & \text { Thickness; thickness of component } k \\ \Delta t^{k} t_{\min }^{k} & \text { Minimum allowable thickness for component } k \\ \mathbf{U} ; \mathbf{u}_{e} & \text { Manufacturability margin for component } k \\ V_{f} & \text { Displacement; displacement for element } e \\ \mathbf{z} & \text { Allowable volume fraction of solid material } \\ \gamma & \text { Decision variable for optimization } \\ \epsilon & \text { Smoothing for bulk density Heaviside function } \\ \phi & \text { Smoothing for edge transition Heaviside function } \\ \bar{\rho} ; \bar{\rho}^{k} & \text { Distance field from morphable components } \\ \rho_{e} & \text { Bulk density; bulk density of component } k \\ \theta & \text { Density of finite element } e \\ & \text { Feature/component angle from build direction }\end{array}$

\section{INTRODUCTION}

Additive manufacturing (AM) has become a compelling alternative to traditional manufacturing technologies for the fabrication of complex, organic shapes produced by topology optimization [1]. The ability of AM to inexpensively produce complex shapes has led to a variety of topology optimization applications including mesostructured or lattice materials $[2,3]$.

Despite the removal of constraints applicable to other manufacturing processes, AM brings with it unique manufacturing considerations [4]. Liu and Ma [1] summarize work applying two kinds of manufacturing constraints from the AM process in topology optimization problems: the minimum feature size (or the smallest element of a design which can be realized by the manufacturing process), and the overhang angle (the maximum degree of overhang achievable by the process without requiring support structures). The complex interaction between feature shape, orientation, and manufacturability typically necessitate overly conservative values for these constraints, restricting the design domain to ensure manufacturability. The AM process is capable of producing some features not permitted by these constraints, and constraints which more accurately reflect the real process capabilities should make more performant designs possible. For many optimization objectives, theoretical work indicates that the optimal designs consist of infinitely many infinitely small members, so the ability to produce smaller members increases the optimality of the design [5].

In previous work, the authors developed a new design constraint for AM processes by experimentally determining minimum feature size as a continuous function of feature shape and build orientation [6,7]. The resulting function can be used to account for both overhang and minimum feature size effects (by realizing a penalty of a large minimum feature size for overhanging features), and captures more closely the real capabilities of the process to produce geometry. Note that these design rules do not consider mechanical capability or surface finish.

In this work this parametric constraint function is applied to the topology optimization problem, presenting a new way of formulating the minimum feature size constraint based on the shape and orientation of each design feature. To accomplish this, the Moving Morphable Component (MMC) framework [8] is adapted to obey the functional feature size constraint and "bootstrapped" (i.e. provided with initial conditions) using the Solid Isotropic Material with Penalization (SIMP) method [9]. The new method is termed the "Bootstrapped Constrained Moving Morphable Components", or bcMMC. 
After a brief literature review, the manufacturing constraint is described and applied to the topology optimization problem, followed by an explanation of the bootstrapping approach. Lastly, preliminary results are presented and discussed.

\section{BACKGROUND}

The topology optimization problem seeks to divide a design domain into solid $(\rho=1)$ and void $(\rho=0)$ regions in order to minimize an objective function; the objective considered here is compliance minimization. Two recent surveys cover the vast literature in this field [10,11], and only a brief introduction to two solution approaches is given here.

In this work, the domain is divided into uniform rectangular finite elements. The problem statement is

$$
\begin{aligned}
\min _{\mathbf{z}}: & f(\mathbf{z}, \mathbf{U})=\sum_{e=1}^{n_{e}} \mathbf{u}_{e}^{T} \mathbf{k}_{e}\left(\rho_{e}(\mathbf{z})\right) \mathbf{u}_{e} \\
& \quad \mathbf{k}_{e}\left(\rho_{\mathbf{e}}(\mathbf{z})\right)=\left[E_{\min }+\rho_{e}^{p}(\mathbf{z})\left(E-E_{\min }\right)\right] \mathbf{k}_{0} \\
\text { subject to : } & \mathbf{K U}=\mathbf{F} \\
& \frac{1}{n_{e}} \sum_{e=1}^{n_{e}} \rho_{e}(\mathbf{z})-V_{f} \leq 0 \\
& \mathbf{z}_{\text {min }} \leq \mathbf{z} \leq \mathbf{z}_{\max }
\end{aligned}
$$

where the state variable $\mathbf{U}$ is the element displacements, each of the $n_{e}$ elements having a stiffness $\mathbf{k}_{e}$ set by its individual density $\rho_{e}$ which is controlled by the decision variable $\mathbf{z}$. The linear elastic relationship is the state equation, where $\mathbf{F}$ is the vector of applied loads and $\mathbf{K}$ the global stiffness matrix. The volume constraint $V_{f}$ restricts the total fraction of solid elements.

In the SIMP approach, the decision variable consists of the continuous density of each element, i.e. $\rho_{e}(\mathbf{z})=z_{e}$ and $\mathbf{z}_{\min }=\mathbf{0} ; \mathbf{z}_{\max }=\mathbf{1}$. Solid/void solutions are encouraged by penalizing the elastic modulus of intermediate-density elements using Eqn. (1b) where $E$ and $E_{\min }$ are the elastic modulus of solid and void elements, respectively; $\mathbf{k}_{0}$ is the fully dense element stiffness matrix; and $p$ is a penalization constant set to 3.0 [9]. The optimized structure is developed implicitly as the decision variable sets the density of each element; post-processing converts this grayscale image to a smooth manufacturable design. To ensure the created structures are accurately modelled by FEA, the filtering approach of Guest et al. [12] is employed to enforce a constant minimum thickness of all solid regions.

\section{Moving Morphable Components}

In contrast to the implicit SIMP approach, the MMC framework defines geometry explicitly, using the decision variable $\mathbf{z}$ to define control points on a set of parametric primitive shapes ("morphable components") which are projected onto the design domain to set element densities [13]. The new approach described later in this paper is an adaptation of the MMC formulation described below, which is itself adapted from Refs. [13-15].

The design domain is pre-populated with components each consisting of the offset of a line segment parameterized by endpoint locations and offset distance (thickness); see Figure 1. The decision variable is constructed by combining the parameters of each component, i.e. $\mathbf{z}=$ $\left[\left(\mathbf{c}^{1}\right)^{\mathrm{T}},\left(\mathbf{c}^{2}\right)^{\mathrm{T}}, \ldots,\left(\mathbf{c}^{k}\right)^{\mathrm{T}}, \ldots,\left(\mathbf{c}^{n_{c}}\right)^{\mathrm{T}}\right]^{\mathrm{T}}$, with the parameter of the $k$ th component $\mathbf{c}^{k}=$ 
$\left[x_{1}^{k}, y_{1}^{k}, x_{2}^{k}, y_{2}^{k}, t^{k}\right]^{\mathrm{T}}$. Let $\phi\left(x, y, \mathbf{c}^{k}\right)$ be the signed Euclidian distance (interior positive) from a point $(x, y)$ on the domain to the component defined by $\mathbf{c}^{k}$, following the approach of Eqn. 4 in Deng and Chen [13].

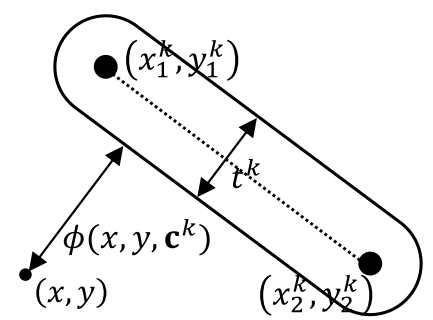

\section{FIGURE 1. SCHEMATIC OF COMPONENT $\boldsymbol{k}$}

To construct the density field, a smoothed Heaviside function is applied to the distance field for each component at each finite element centroid, according to

$$
H(\phi, \epsilon, \bar{\rho})= \begin{cases}\bar{\rho} & \text { if } \phi>\epsilon \\ \frac{3 \bar{\rho}}{4}\left(\frac{\phi}{\epsilon}-\frac{\phi^{3}}{3 \epsilon}\right)+\frac{\bar{\rho}}{2} & \text { if }-\epsilon \leq \phi \leq \epsilon \\ 0 & \text { otherwise }\end{cases}
$$

with parameters $\phi$, the distance to the component (arguments to $\phi$ omitted for clarity); $\epsilon$ the regularization parameter describing the width of the transition; and $\bar{\rho}$, the bulk density of the component (i.e. 1.0). In this way elements smoothly transition from void (outside) to $\bar{\rho}$ (inside) over a band of size $2 \epsilon$. The density of FEA element $e$ (denoted $\rho_{e}$ ) is assigned by sampling this function at the element centroid $\left(x_{e}, y_{e}\right)$ for each component and retaining the maximum:

$$
\begin{aligned}
\rho_{e}(\mathbf{z}) & =\max _{k=1 . . . n_{c}} H\left(\phi\left(x_{e}, y_{e}, \mathbf{c}^{k}\right), \epsilon, \bar{\rho}\right) . \\
\text { Note that } \mathbf{z} & =\left[\left(\mathbf{c}^{1}\right)^{\mathrm{T}},\left(\mathbf{c}^{2}\right)^{\mathrm{T}}, \ldots,\left(\mathbf{c}^{n_{c}}\right)^{\mathrm{T}}\right]^{\mathrm{T}}, \bar{\rho}=1
\end{aligned}
$$

Unlike SIMP, the design variable $\mathbf{z}$ consists of the parameters of all the components; as a result the minimum and maximum bounds on $\mathbf{z}$ are more complex, dependent on the domain shape and range of acceptable element thicknesses.

Derivatives are computed using the chain rule. Equations (1a) and (1b) provide the derivative of $f$ with respect to element density $\rho_{e}$; the derivative with respect to the decision variables $\mathbf{z}$ can be obtained as

$$
\begin{aligned}
& \frac{\partial f}{\partial z_{j}}=\sum_{e=1}^{n_{e}} \frac{\partial f}{\partial \rho_{e}} \frac{\partial \rho_{e}}{\partial z_{j}} \\
& \frac{\partial f}{\partial \rho_{e}}=-p \rho_{e}^{p-1}\left(1-E_{\min }\right) \mathbf{u}_{e}^{T} \mathbf{k}_{0} \mathbf{u}_{e}
\end{aligned}
$$

For implementation convenience, the derivative of each density with respect to each design variable is computed numerically, following the approach of Refs. [13,15]. This derivative could be constructed explicitly by replacing the max function in Eqn. (3) with a "softmax" alternative. 
In this work when reporting and comparing objective function values, the "thresholded compliance" will be used, denoted $\bar{f}$ and constructed by thresholding the densities in the design domain so as to satisfy the volume constraint. This eliminates artifacts due to penalization of intermediate density elements.

\section{Bootstrapping Approaches}

"Bootstrapping" refers to the idea of leveraging existing resources to produce a more complex or capable outcome. Here, bootstrapping refers to using SIMP to select the starting locations for components in the MMC process, described in detail shortly. Similar concepts have been explored by Bremicker et al. [16], who extracted a topology from a SIMP solution and used it as the starting point for shape optimization, leveraging a computer vision technique to produce a shape skeleton, or single-pixel-wide backbone of a shape [17]. Similar chaining of topology and subsequent shape optimization are used under human direction in engineering practice, for an example see Chang and Tang [18].

In a recent review, Sigmund and Maute [11] point out the need for more topology optimization research in "hybrid approaches" in which the benefits of two topology optimization approaches are combined to remove initial condition sensitivity and improve convergence. While not the primary contribution of this work, the approach presented here also begins to address this need.

\section{Manufacturing Constraints in Topology Optimization}

Liu and Ma [1] review various approaches for incorporating manufacturing constraints into the topology optimization problem in order to avoid post-processing steps which often destroy the local optimality of the solution. For AM, two most-studied constraints are the minimum feature size and the maximum overhang angle.

Minimum feature size has long been integrated into the SIMP approach in order to produce mesh-dependent designs (i.e. through filtering the density field), and Lazarov et al. [19] survey these approaches in the context of manufacturability. Recently, an approach which adapts this filter to the direction-dependent feature size characteristic of most AM processes has also been presented [20]. In the MMC framework, minimum feature size for each component can be explicitly constrained, but narrow hinges can still form where two members overlap just slightly, as explored by Hoang and Jang [21].

Overhang angle has also been studied, but far less extensively [22-25]. Some authors utilize asymmetric filters and projections in a framework similar to SIMP, to ensure that solid elements are only allowed in locations with appropriate support leading to optimized structures containing many small elements which simultaneously bear load and support otherwise unmanufacturable structures $[22,23,25]$. Typically, these approaches incur a penalty to the objective function of 2$10 \%$ when applied to standard test problems.

Guo et al. [24] built specific constraints into the formulation of their MMC and Moving Morphable Voids (MMV) approaches to ensure the result follows the overhang angle constraint. This works well in their MMC implementation, in which the orientation of the part on the build plate is included as a decision variable and results in only $1 \%$ increase in the objective function compared to an unconstrained design. Their MMV approach does not include orientation as a decision variable and the resulting geometry differs significantly from the optimal, with a corresponding 14\% penalty in the objective function. Liu and Ma [1] cite overhang angle constraints as a significant unsolved problem in topology optimization for AM, noting the substantial change in shape and objective function value seen in most approaches. 


\section{DATA-DRIVEN MANUFACTURING CONSTRAINTS}

In this section, the conceptual basis for the proposed additive manufacturing constraint is presented. In previous work [6,7], a piecewise-continuous, polynomial minimum feature size design rule function for small features produced by an AM platform was presented. The design rule returns the minimum manufacturable diameter of a beam given its length and angle from the build direction; see Figure 2.

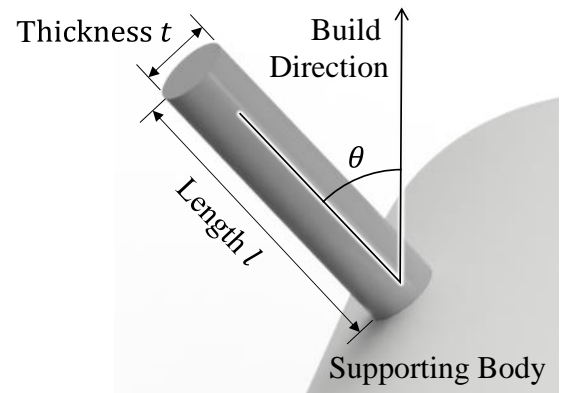

FIGURE 2. SCHEMATIC OF A SMALL FEATURE

Instead of imposing an explicit overhang constraint, the experiment used required all manufacturable features to possess a continuous core of solid material. Because overhanging features in the process studied exhibit surface defects in the downward direction, correspondingly larger minimum feature thicknesses were obtained, suitably penalizing these difficult-tomanufacture features. The experimental data collected also includes horizontal "bridging" features which connect two supporting bodies. This ability to incorporate bridges with experiment-driven sizing information is a significant advantage of the present approach over overhang angle constraints, as it allows some manufacturable horizontal features in the final design.

For the present work, the 2D optimized structure is assumed to extend a relatively small distance into the plane, and manufacturing constraints from small cylinders are assumed valid for the rectangular cross-section of the $2 \mathrm{D}$ features extended uniformly into the plane. The experimentally-determined constraint function for a material extrusion machine, including bridging data, are shown in Figure 3 and denoted $S(l, \theta)$ where $l$ is the feature length and $\theta$ is the angle between the feature axis and the build direction. This manufacturability constraint function is assumed to be already known at the start of the optimization process, and is not derived in this work.

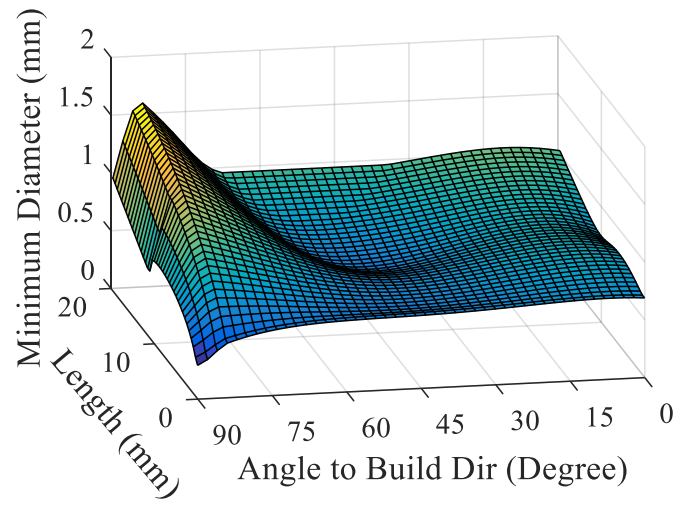

FIGURE 3. THE PARAMETRIC CONSTRAINT FUNCTION 


\section{BOOTSTRAPPED CONSTRAINED MOVING MORPHABLE COMPONENTS}

In this section, the details of the bcMMC approach are presented, which builds on the MMC method described above. Specific extension to the MMC method are described, including the integration of the data-driven manufacturing constraint and the bootstrapping method used. After a brief overview, subsections present details of the bootstrapping process and the constrained MMC method.

\section{Overview}

Figure 4 shows an overview of the bcMMC algorithm, which consists of two phases, an initial bootstrapping phase and a main constrained optimization phase. It takes as input an optimization domain, load and support conditions, and a build direction (Figure 4a). In the bootstrapping phase, a SIMP solution with fixed minimum feature size is computed (Figure $4 \mathrm{~b}$ ). Then, the density field is converted into a set of connected morphable components (Figure 4c) which serve as the starting configuration for the constrained optimization. This is described in the Phase 1 subsection below.

In Phase 2, the MMC optimizer adjusts the component endpoints and thicknesses to minimize the objective (Figure 4d). At each iteration, the minimum manufacturable thickness for each component is assessed using the constraint function described previously (Figure 4e). As the thickness of each component approaches the minimum manufacturable value, its bulk density is smoothly reduced from fully dense to void. Each component is projected to the new density field (Figure 4f), FEA and sensitivity calculations are performed, and the optimizer updates the decision variables (Figure 4g). The process repeats until a convergence criterion is reached and is described in detail in the "Phase 2" subsection.

For example consider the component labelled with a triangle in Figure 4d. On each iteration, the component's orientation and length and thickness are used to place it in the manufacturability space, which assigns it a "manufacturability margin" based on the difference between the current thickness (triangle in Figure 4e) and the constraint surface. In this case, the triangle is near the constraint surface and marginally manufacturable, so it is assigned an intermediate bulk density, which is used when the component is projected onto the FEA element grid (Figure 4f).

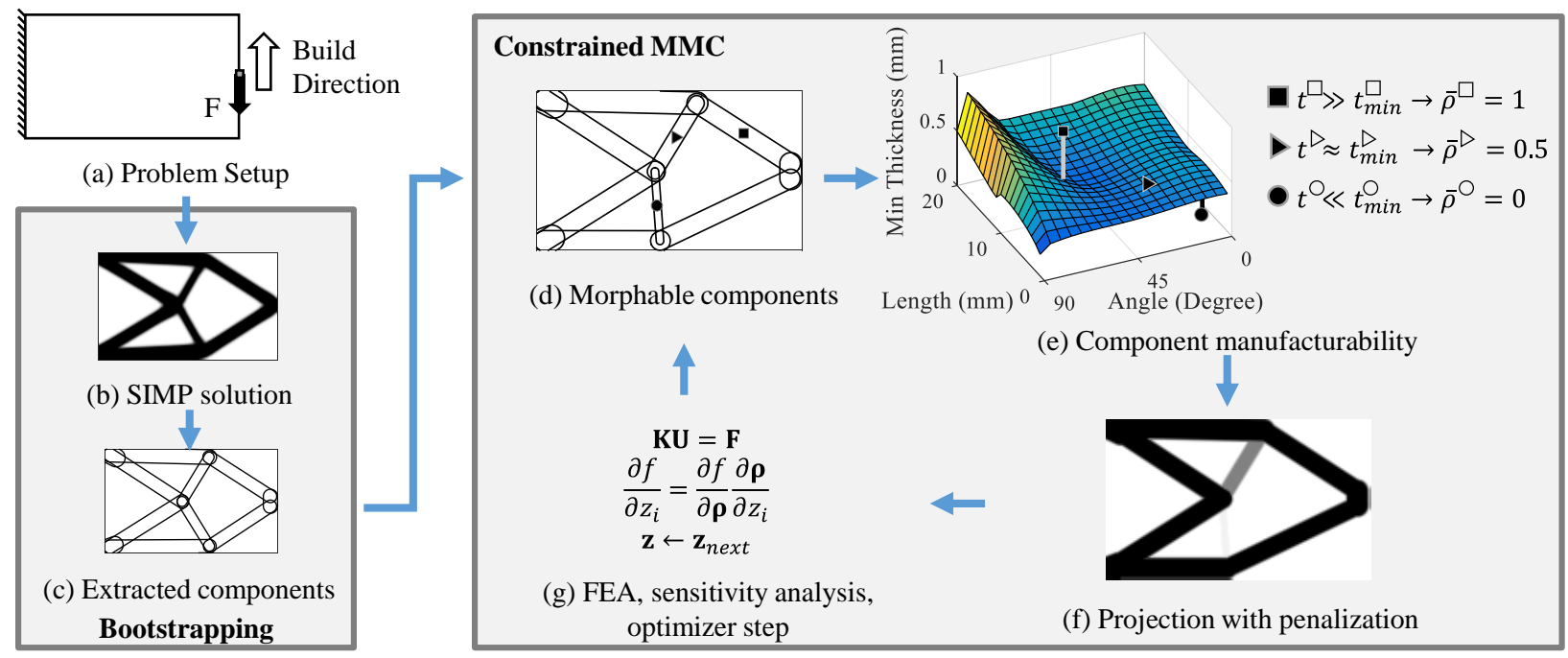

FIGURE 4. FLOWCHART OF THE bCMMC PROCESS; SEE TEXT FOR DESCRIPTION 


\section{Phase 1: Bootstrapping}

In this subsection, the bootstrapping approach which provides the main optimizer with an intelligent and connected initial component configuration is detailed. The SIMP algorithm is used to provide a preliminary component layout for the constrained optimization procedure described in the next subsection, producing a "bootstrapped constrained" MMC or bcMMC approach. Bootstrapping provides a dramatic improvement in convergence time to a stable equilibrium compared with an MMC solution initialized with arbitrarily-distributed components (measured in both compute time and iteration count). However, the search space is narrowed by starting the MMC optimization near a local minimum without the opportunity to explore different and possibly more performant areas of the search space. Note that the main algorithm can still effect topology changes by overlapping or penalizing members which it deems inefficient in light of the manufacturing constraints. This procedure follows in the spirit of Bremicker et al. [16], who used the shape skeleton as a means to extract features from a SIMP solution.

Here, the SIMP algorithm is first run for a small number of iterations to produce an initial solution to the optimization problem under a fixed minimum feature size that is just less than the smallest manufacturable feature (see Figure 5a). SIMP quickly converges from a uniform initial condition (i.e. gray; not shown) to near a local optimum, reducing the need for the MMC algorithm to slowly move components over long distances.

To create the initial component layout, the SIMP density field is first preprocessed by applying a threshold to the grayscale design and using a morphological closing operation to eliminate small holes (Figure 5b). The shape skeleton is then computed (Figure 5c). By design, the shape skeleton preserves the topology of the input, but converts all members to single-pixel-wide features. The junction points in the skeleton, where two or more skeletal branches come together, are extracted.

Adjacent junction points in the skeletal graph are connected with straight segments (Figure 5d). Each segment will form the center line of a component. Segments which are shorter than a pre-determined threshold, $l_{\text {min }}$, are removed and nearby junction points merged. In order for each linear component to more precisely reflect the original shape, the locations of all junction points are moved so as to minimize the deviation of the linear segments from the center lines of the features in the density field (Figure 5e). In addition, new component segments are added to connect the skeleton to each of the load or support points in the problem setup, ensuring that the design does not become disconnected from the boundaries.

To provide the optimizer with additional flexibility in determining the shape and structure of the final design, long components (in this case those with length greater than $5 l_{\text {min }}$ ) are divided in half, with a new junction point inserted at the midpoint and initial thicknesses set according to the average thickness of the corresponding region of the SIMP design, scaled so as to respect the volume constraint (Figure 5f). 


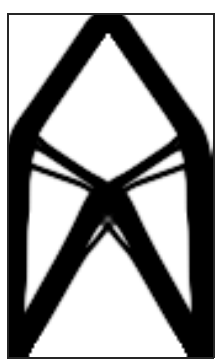

(a)

SIMP density field

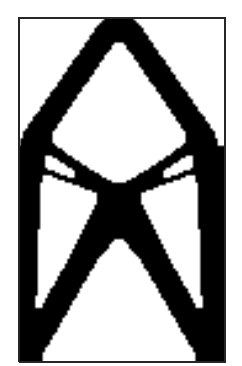

(b)

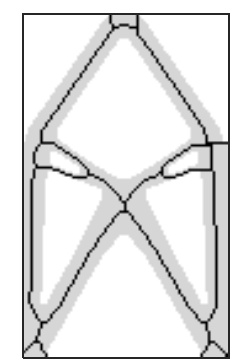

(c)

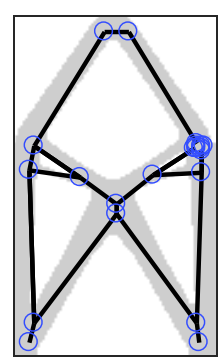

(d)

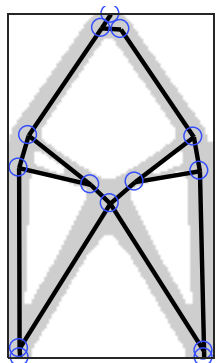

(e)

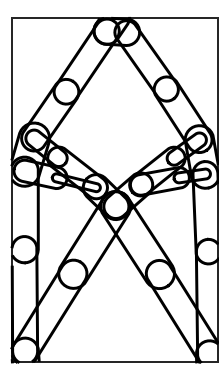

(f)

Edge Extraction Junction Adjustment Component Layout

\section{FIGURE 5. BOOTSTRAPPING PROCEDURE, ILLUSTRATED; SEE TEXT FOR DESCRIPTION}

\section{Phase 2: Constrained MMC Optimization}

Two key modifications of the MMC framework are presented to enforce the manufacturing constraint: (1) the components are explicitly and inexorably connected, and (2) the density of unmanufacturable features is penalized.

For reasons described later, it is desirable to explicitly link the end points of each component to its neighbors. The bootstrapping process provides this linkage information, and the structure of the decision variable $\mathbf{z}$ is changed from that presented in the Background to consist of a series of junction point coordinates followed by a series of component thicknesses, while the mapping between junction points and thicknesses is tracked separately. This is similar to the Connected Morphable Components of Ref. [13]; however, in the present work more than two components can share a junction.

At each update in the new bcMMC framework, the $k$ th component is assigned a minimum thickness value, $t_{\text {min }}^{k}=t_{\text {min }}\left(\mathbf{c}^{k}\right)=S\left(l^{k}, \theta^{k}\right)$ based on its length $l^{k}$ and orientation $\theta^{k}$, which are computed from the component endpoints. Components with "manufacturability margin" $\left(\Delta t^{k}\right.$, defined as $t^{k}-t_{\text {min }}^{k}$ ) less than zero cannot be produced and should not be permitted by the optimizer. The build direction is specified manually.

This manufacturability information is integrated into the bcMMC method by penalizing the component density of unmanufacturable features. Because the optimization formulation already penalizes intermediate-density elements (using $p$ in Eqn. (1b)), the optimizer is incentivized to either bring them back to full density (by thickening them or changing the angle/shape of the feature) or shrink them until they reach zero density, removing them from the solution. Density penalization based on member thickness is used successfully by Norato et al. [14], where noninteger components were penalized so that the design could be realized using readily-available stock material. A penalization approach has the additional benefit of allowing the optimizer to remove components from the problem by exploiting the penalization to assign them zero density, something difficult in other MMC implementations.

The bulk density of a component is controlled by specifying the maximum value of the smoothed Heaviside projection, i.e. by setting $\bar{\rho}$ in Eqn. (3). Reducing $\bar{\rho}$ from unity causes the entire interior of a component to become less dense (see Figure 4f). Ideally, the bulk density of the component should transition from solid to void as the manufacturability margin drops to zero. To avoid discontinuities, the transition is governed by another application of the smoothed Heaviside function, this time with (new) smoothing parameter $\gamma$. Equation (3) can be rewritten 


$$
\begin{aligned}
& \rho_{e}(\mathbf{z})=\max _{k=1 . . . c_{c}} H\left(\phi\left(x_{e}, y_{e}, \mathbf{c}^{k}\right), \epsilon, \bar{\rho}^{k}\right) \\
& \bar{\rho}^{k}=H\left(\Delta t^{k}, \gamma, 1\right) \\
& \Delta t^{k}=t^{k}-t_{\min }\left(\mathbf{c}^{k}\right)=t^{k}-S\left(l^{k}, \theta^{k}\right)
\end{aligned}
$$

Note that a formula for explicit derivatives could still be obtained, despite the added interaction between $\bar{\rho}^{k}$ and $\mathbf{z}$, because the constraint function $S$ is differentiable. Numeric derivatives are used in this implementation, however.

The above approach handles manufacturability constraints for individual components. However, components often overlap to form larger effective structures in MMC solutions. Since longer features require larger minimum diameters to be manufacturable, two individually manufacturable features could join end to end to create an unmanufacturable result. To overcome this difficulty, component endpoints are explicitly merged in the bcMMC approach, producing "chains" of components linked end-to-end to create a larger equivalent feature that can also be evaluated for manufacturability.

As a heuristic to prevent chains from forming unmanufacturable features, the components in the chain are combined into an "equivalent component" created by taking the average angle, average thickness, and total length of the chain elements (see Figure 6). A thickness margin for the equivalent chain is obtained by querying the constraint function using the equivalent component's shape parameters. The bulk density $(\bar{\rho})$ for all components in a chain is redefined to use the smallest of the thickness margins of each chain component and of the equivalent component. This causes entire chains to be removed all at once from the design space, preventing dangling components which are not supported from forming.

Experimental evidence suggests a continuation approach which slowly decreases the smoothing parameter $\gamma$ over the first 40-80 iterations allows the optimizer time to thicken components before they are reduced to zero density. Such an approach is used here.

One additional minor implementation detail is of note: To avoid having the optimizer exploit the volume constraint by placing part of a component (partly) outside the analysis domain, the locations of the junction points are required to remain away from the domain boundary, except on edges with symmetry boundary conditions and on the build plate (geometry on the build plate is assumed to be more easily producible because it is everywhere supported). This "Endpoint Keepout Zone" is specified in the problem definition and works to keep most of the component mass inside the domain, so that the FEA, volume constraint, and manufacturability assessment can all be applied uniformly (see Figure 7). It does not add any effectiveness to the bcMMC approach, only avoids unrealistic results.

\section{RESULTS}

In this section, some preliminary results obtained using the bcMMC approach are presented. First, a validation study evaluates the bootstrapping approach, followed by two examples demonstrating the value of using feature-driven manufacturability penalties. Unless otherwise stated, parameters for all problems in this section are shown in Table 1, where $w_{e}$ and $w_{d}$ correspond to the width of an element and of the domain, respectively. The optimizer used is the Method of Moving Asymptotes algorithm by Svanberg [26]; SIMP results are generated using the top 110 code by Andreassen et al. [27] utilizing the projection filter from [12]. 


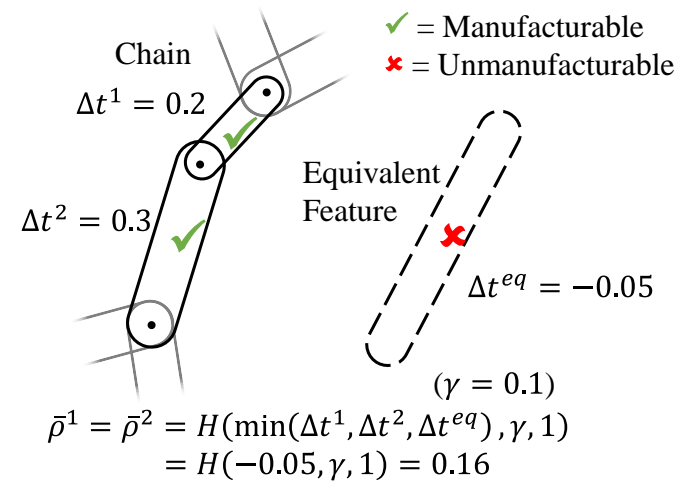

FIGURE 6. EXAMPLE OF CHAINED COMPONENTS

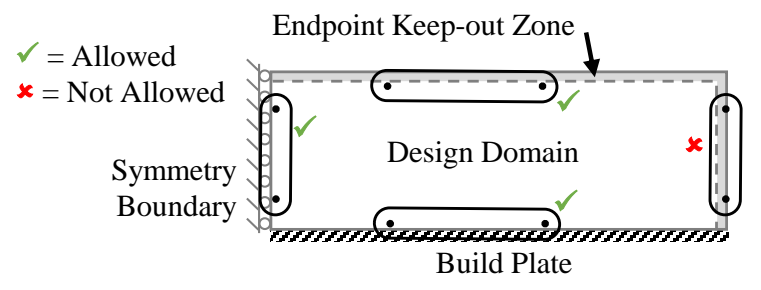

FIGURE 7. ENDPOINT KEEP-OUT ZONE

TABLE 1. DEFAULT PARAMETERS

\begin{tabular}{cc}
\hline Parameter & Value \\
\hline \hline Fully dense elastic modulus $E$ & 1 \\
\hline Void elastic modulus $E_{\min }$ & $10^{-9}$ \\
\hline Poisson's ratio $v$ & 0.3 \\
\hline Intermediate density penalty $p$ & 3 \\
\hline SIMP iterations before bootstrapping & 50 \\
\hline Heaviside smoothing for edges $\epsilon$ & $0.5 w_{e}{ }^{\mathrm{a}}$ \\
\hline Initial smoothing for bulk penalization $\gamma_{0}$ & $0.1 w_{d}{ }^{\mathrm{b}}$ \\
\hline Final smoothing for bulk penalization $\gamma_{f}$ & $0.008 w_{d}{ }^{\mathrm{b}}$ \\
\hline Steps used in the continuation of $\gamma$ & 60 \\
\hline MMC Derivative step size, $x$ and $y$ & $2 w_{e}{ }^{\mathrm{a}}$ \\
\hline MMC Derivative step size, $t$ & $0.2 w_{e}{ }^{\mathrm{a}}$ \\
\hline$l_{\text {min }}$ used in bootstrapping & 0.1 mm $(0.039$ in) \\
\hline${ }^{\mathrm{a}} w_{e}$ refers to the width of an element (in domain units) \\
${ }^{\mathrm{b}} w_{d}$ refers to the width of the domain (in domain units)
\end{tabular}

\section{Convergence of bcMMC}

Because SIMP quickly provides an initial configuration close to a local minimum, the bootstrapping approach is expected to converge more quickly to a good solution. To evaluate this 
consider compliance minimization in the 160x80 element domain (2:1 aspect ratio) shown in Figure 8a. It is solved using three approaches: (1) Using only SIMP; (2) using only the main MMC framework with a set of isolated components in the initial condition shown in Figure $8 \mathrm{~b}$ (as in [15]); and (3) using the bcMMC technique with 50 SIMP iterations. The volume fraction is set to $40 \%$, and the minimum feature size is set to 2.4 element widths. For this trial, the keep-out zone is disabled and no manufacturability penalties are applied.

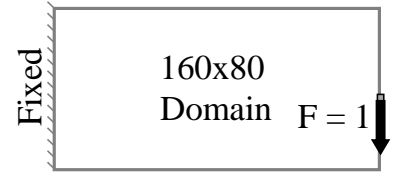

(a)

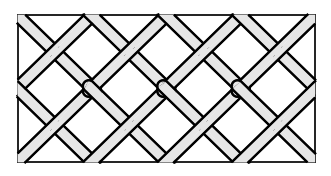

(b)

\section{FIGURE 8. (a) DESIGN DOMAIN (b) INITIAL COMPONENT DISTRIBUTION}

Summary results are presented in Table 2. The iteration history plot (shown in Figure 9) shows the SIMP result quickly converges to a low (good) objective function value, beyond which slow, steady improvement is seen. The non-bootstrapped MMC solution converges slowly due to the need to move each individual component into position, but ultimately reaches an objective function value 5.4\% higher than SIMP. The bootstrapping process creates an initial component layout after 50 iterations of SIMP, after which a few iterations of higher objective are seen as the optimizer adjusts for irregularities introduced by the bootstrapping process, but then drops quickly to an objective function value only about $1.6 \%$ higher than SIMP, stopping after only 146 MMC iterations. The optimization is stopped when the largest iteration-to-iteration change in any entry in the state vector $\mathbf{z}$ drops below $0.1 \%$ of its allowable range.

To quantitatively assess the differences in convergence, the number of iterations required for thresholded compliance to drop and remain below 80 is considered (see dashed line in Figure 9). The bcMMC solution reaches this threshold in 70 iterations, which though worse than SIMP is a $72 \%$ improvement over non-bootstrapped MMC. SIMP consistently outperforms unconstrained bcMMC; however SIMP is unable to incorporate the feature-driven manufacturing constraints which are the focus of this work.

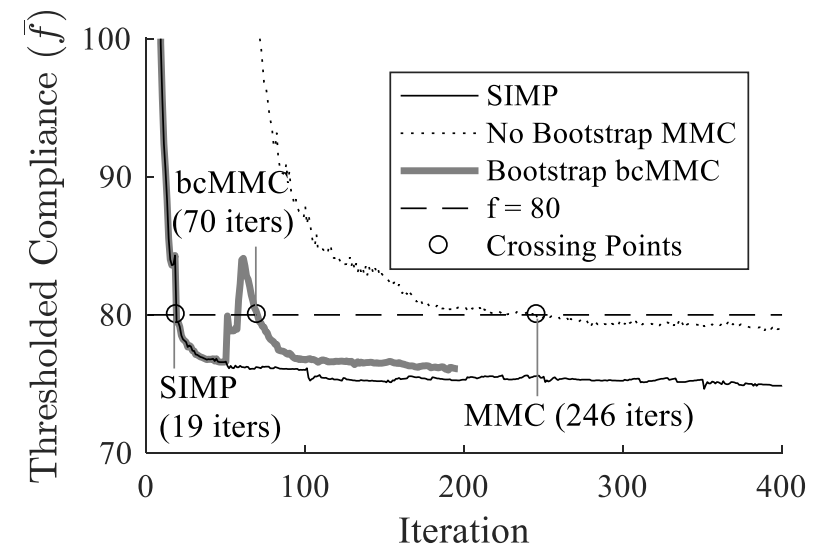

FIGURE 9. BOOTSTRAPPING TEST CONVERGENCE; LOWER VALUES ARE BETTER 
TABLE 2. BOOTSTRAPPING TEST RESULTS

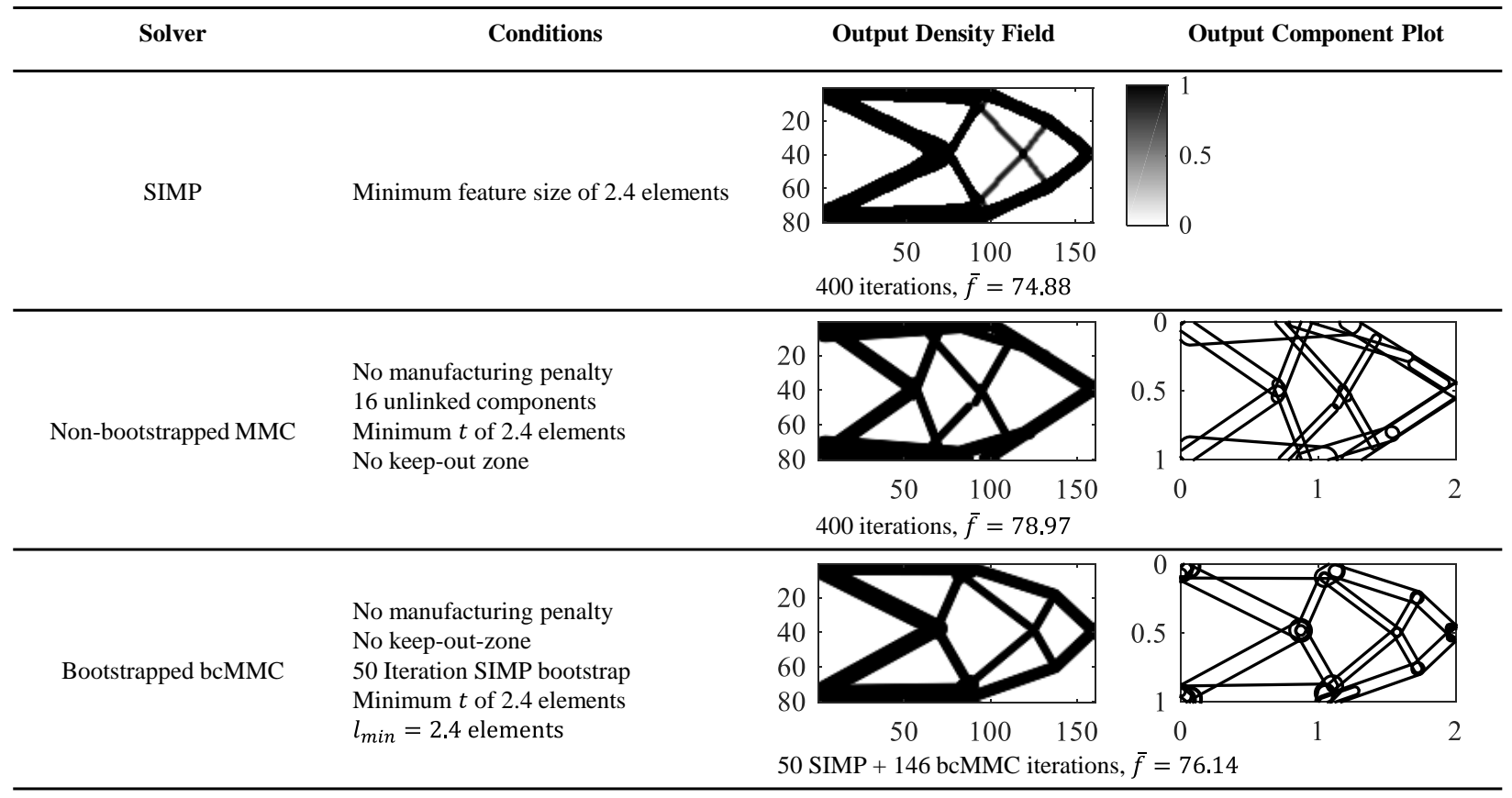

\section{The Short Beam Problem}

The short beam problem considers the design of a manufacturable cantilevered beam with the configuration shown in Figure 10. The 200x100 FEA domain measures $40 \mathrm{~mm}$ by $20 \mathrm{~mm}(1.57 \mathrm{in}$ by 0.79 in). The build plate and keep-out zone definition for this problem are also shown in Figure 10. The SIMP filter size is set to create $0.6 \mathrm{~mm}(0.024 \mathrm{in})$ minimum features, a thickness which is just below the smallest value for any feature at any orientation or length in the manufacturing constraint function for the process used. The volume fraction is set to $50 \%$. The SIMP algorithm is run for 50 iterations prior to bootstrapping, and the main optimization code allowed to run until convergence up to 200 additional iterations.

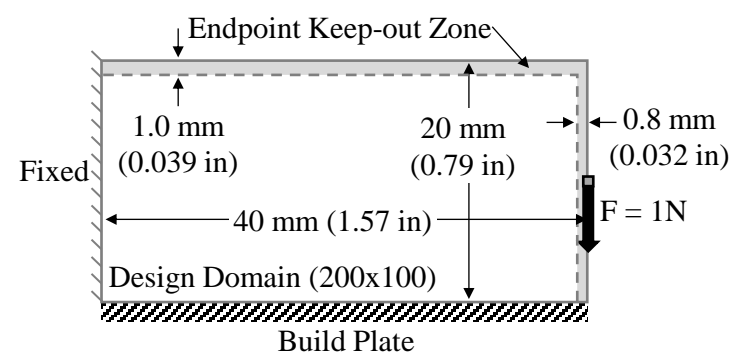

\section{FIGURE 10. SETUP FOR THE SHORT BEAM PROBLEM}

Intermediate results for the bootstrapping are shown in Figure 11. After 50 iterations, the SIMP code produces geometry with fine details (Figure 11a), but some are lost in the conversion to component layout shown in Figure 11b. The small features which are removed have a relatively minor impact on the overall performance of the structure, and are likely not manufacturable anyway. 


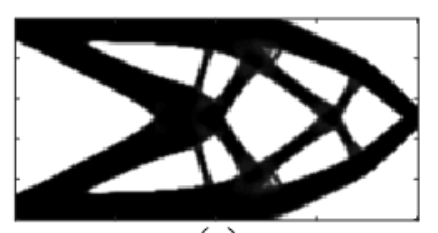

(a)

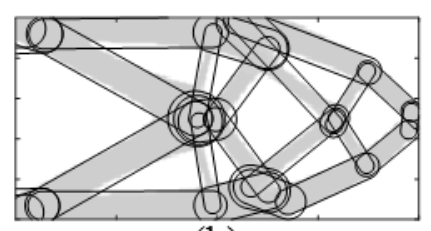

(b)

\section{FIGURE 11. BOOTSTRAPPING FOR THE SHORT BEAM PROBLEM (a) SIMP RESULT (b) COMPONENT LAYOUT}

Table 3 show the bcMMC layouts and density fields for two different build directions. In both cases, the manufacturability penalty is active. In the top row, the build direction is orthogonal to the build plate shown in Figure 10. All of the small features present in the SIMP design are retained, though some are reshaped, resulting in a thresholded compliance $1.2 \%$ higher than a reference SIMP solution with minimum feature size of $1.0 \mathrm{~mm}(0.039 \mathrm{in}$, large enough to generally be manufacturable on the selected process for overhang angles less than $45^{\circ}$ ). Note that in contrast to the reference SIMP solution, the results of both bcMMC solutions presented in Table 3 fully satisfy the parametric minimum feature size design rule and should be manufacturable. Even though many overhanging members exist in this configuration, they all lie above the experimentally-determined minimum feature diameter for their respective orientations.

In the second row of Table 3, the same problem is solved, with the build direction rotated $90^{\circ}$. In this case, most components are more easily manufactured, except for two nearly-horizontal components (see arrows) which have been removed as inefficient by the optimizer by thinning until the effective density is zero and redistributing their material to other components. The objective function value for this case, $\bar{f}=62.55$, lags the SIMP solution by $1.45 \%$. The central large features in this design show some asymmetry due to sensitivity of the shape skeleton operation used in bootstrapping which resulted in uneven penalization of the two horizontal components, and is a clear shortcoming of this approach. Several intermediate steps from this case are shown in Figure 12.

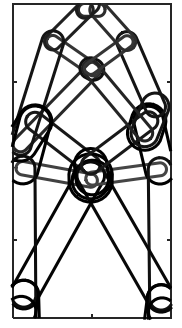

Iteration 10 $\bar{f}=917.53$

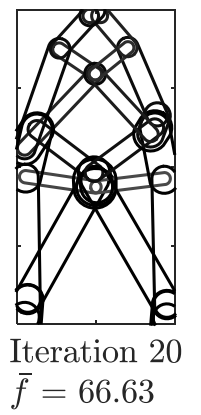

$\bar{f}=66.63$

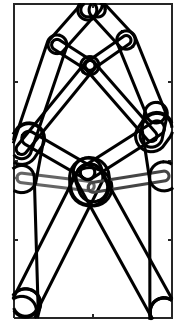

Iteration 60 $\bar{f}=63.27$

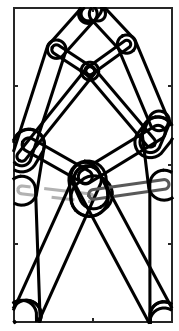

Iteration 100 $\bar{f}=62.35$

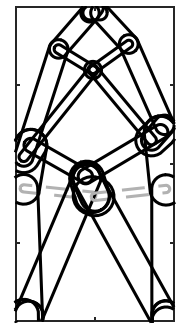

Iteration 140 $\bar{f}=62.38$

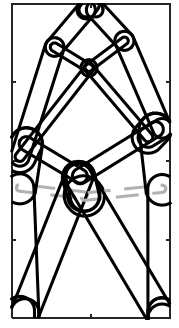

Iteration 180

$\bar{f}=62.46$

\section{FIGURE 12. INTERMEDIATE RESULTS FOR THE ROTATED SHORT BEAM PROBLEM.}

\section{The MBB Beam Problem}

The well-known MBB problem is also solved; Figure 13 shows half of the symmetric design domain modelled by $225 \times 75$ FEA elements and measuring $45 \mathrm{~mm}$ by $15 \mathrm{~mm}$ (3:1). The SIMP implementation used for bootstrapping employs a $p$-continuation approach which yields the more intricate microstructure in the center of the domain by incrementing $p$ from 1.0 to 3.0 in steps of 0.1 every 50 iterations. After $p$ reaches 3.0, the beta parameter of the Heaviside projection filter of 
Guest et al. [12] is incremented until either convergence is reached or when 1200 iterations have occurred. The SIMP filter ensures at least $0.6 \mathrm{~mm}(0.024$ in) thick features, which is just less than the smallest manufacturable feature size. The volume fraction is set to $40 \%$. Because of the continuation used, bootstrapping is performed after all 1200 SIMP iterations, producing the result shown in Figure 14.

TABLE 3. SHORT BEAM PROBLEM RESULTS

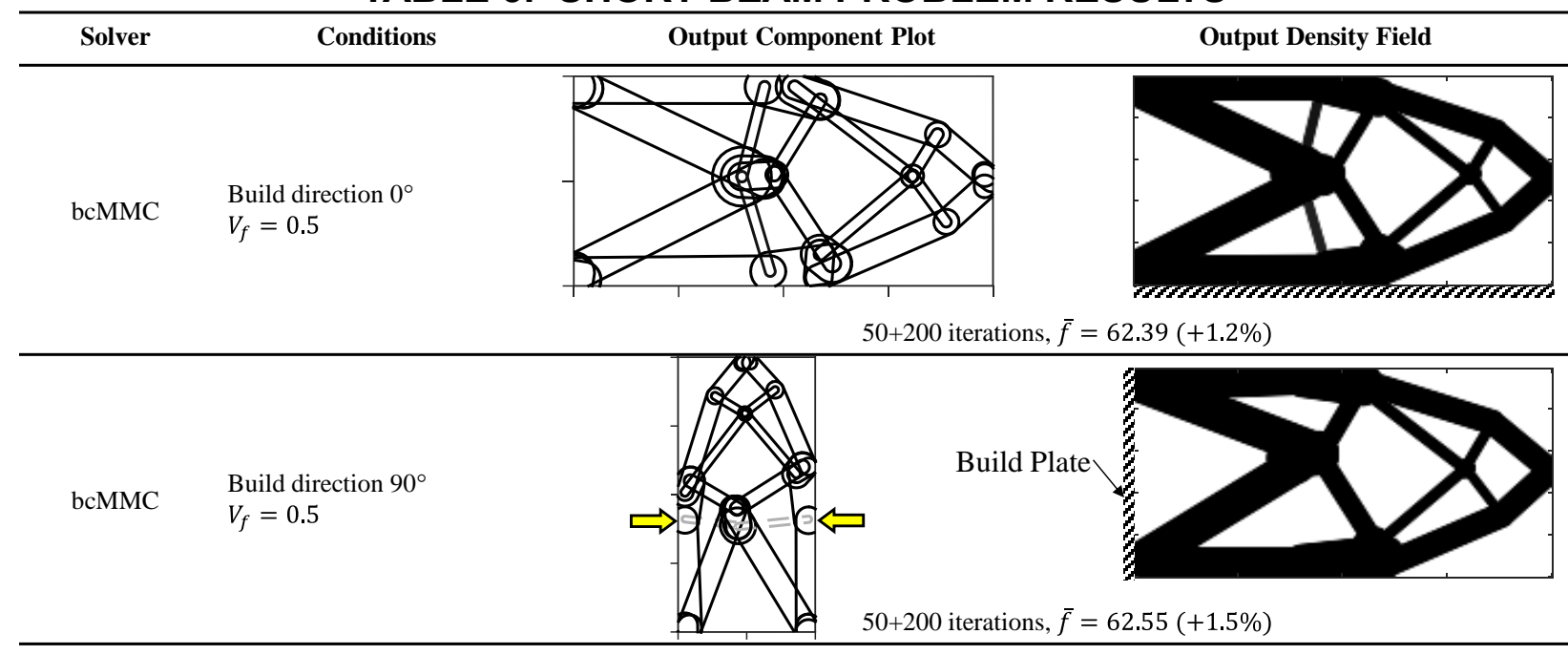

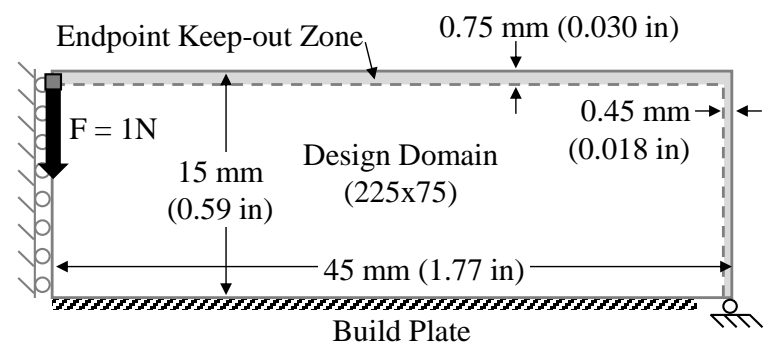

FIGURE 13. SETUP FOR THE MBB PROBLEM

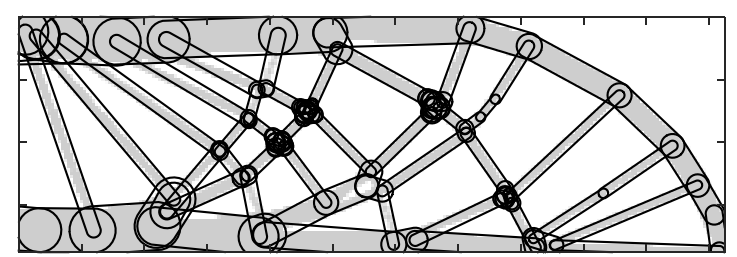

\section{FIGURE 14. SIMP SOLUTION FOR BOOTSTRAPPING IN THE MBB PROBLEM, WITH COMPONENTS OVERLAID}

Table 4 shows three manufactured designs. All three struggle with surface finish in the overhanging features; however the drooping in the very thin members in the more aggressive SIMP case (with minimum member thickness of $0.71 \mathrm{~mm}$ or $0.028 \mathrm{in}$ ) result in a significant fraction of each beam not being fully manifested. The bcMMC optimizer adjusts the overhang angle and thickness of major features in the design to be better supported, and thickens some of the smaller elements to increase manufacturing performance (see arrows in Table 4). The ability of the 
manufacturing constraint to allow bridging members enables the top element to be retained without additional supports, unlike the other overhang topology optimization approaches surveyed.

TABLE 4. MBB PROBLEM RESULTS

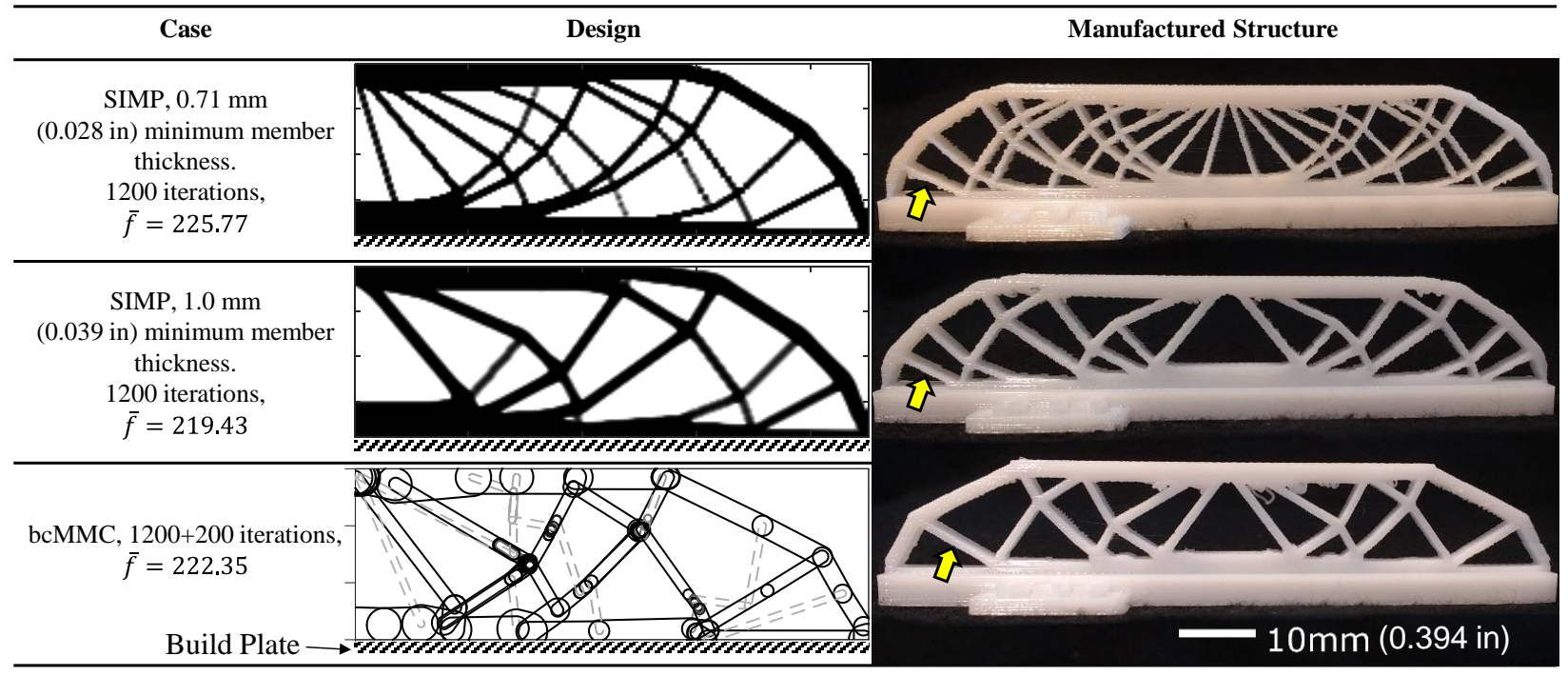

\section{CONCLUSION}

A data-driven constraint function for topology optimization of designs for AM developed previously has been incorporated into a Moving Morphable Components (MMC) topology optimization framework. To support this constraint, which provides a different minimum member thickness based on the shape and orientation of each straight segment in a design, a variant of the MMC framework is presented which utilizes SIMP to generate preliminary designs to serve as starting points. This "Bootstrapped Constrained MMC" approach (bcMMC) implements this manufacturability constraint and provides faster convergence to optimized solutions than reference MMC implementations.

Compared to other manufacturing constraints on topology optimization for AM, which typically utilize a single fixed minimum feature size and a maximum overhang angle, this functional constraint method helps take better advantage of the space of manufacturable geometries, increasing the design complexity the optimizer can achieve. For example, short horizontal "bridging" features can be included, providing solutions which more nearly match the unconstrained case while still being manufacturable.

The compliance minimization examples presented use a constraint function created for a material extrusion AM platform, but the approach is easily generalized to other objective functions and AM processes. The modified optimization process ensures the design is both optimized and manufacturable, and makes relatively small changes to the part topology, component orientation, and component size compared to fixed minimum feature size techniques, with a penalty to the objective function of less than $5 \%$.

Exciting possible extensions of this work include adapting the approach to 3D problems, different objective functions, and other AM processes. Automatically generating and incorporating additional components to act as support material for overhanging features would also be advantageous. 


\section{REFERENCES}

[1] Liu, Jikai, and Ma, Yongsheng. "A Survey of Manufacturing Oriented Topology Optimization Methods" Advances in Engineering Software Vol. 100 (2016): pp. 161-175. DOI

10.1016/j.advengsoft.2016.07.017.

http://linkinghub.elsevier.com/retrieve/pii/S0965997816302174.

[2] Coelho, Pedro, Cardoso, João, Fernandes, Paulo, and Rodrigues, Hélder. "Parallel Computing Techniques Applied to the Simultaneous Design of Structure and Material" Advances in Engineering Software Vol. 42 No. 5 (2011): pp. 219-227. DOI

10.1016/j.advengsoft.2010.10.003.

http://linkinghub.elsevier.com/retrieve/pii/S0965997810001237.

[3] Gu, X.J., Zhu, Jihong, and Zhang, W.H. "The Lattice Structure Configuration Design for Stereolithography Investment Casting Pattern Using Topology Optimization" Rapid Prototyping Journal Vol. 18 No. 5 (2012): pp. 353-361. DOI 10.1108/13552541211250355.

http://www.emeraldinsight.com/doi/10.1108/13552541211250355.

[4] Vayre, Benjamin, Vignat, Frédéric, and Villeneuve, François. "Designing for Additive Manufacturing" Procedia CIRP Vol. 3 (2012): pp. 632-637. DOI 10.1016/j.procir.2012.07.108. http://linkinghub.elsevier.com/retrieve/pii/S2212827112002806.

[5] Zhou, M., and Rozvany, G.I.N. "The COC Algorithm, Part II: Topological, Geometrical and Generalized Shape Optimization" Computer Methods in Applied Mechanics and Engineering Vol. 89 No. 1-3 (1991): pp. 309-336. DOI 10.1016/0045-7825(91)90046-9. http://linkinghub.elsevier.com/retrieve/pii/0045782591900469.

[6] Weiss, Benjamin, Diegel, Olaf, Storti, Duane, and Ganter, Mark. "A Process for Estimating Minimum Feature Size in Selective Laser Sintering” Rapid Prototyping Journal Vol. 24 No. 4 (2018):

[7] Weiss, Benjamin, 2017, “Development of a Process for Determining Minimum Feature Size in Additive Manufacturing with Applications to Topology Optimization" University of Washington, PhD Dissertation, URI http://hdl.handle.net/1773/40934.

[8] Guo, Xu, Zhang, Weisheng, and Zhong, Wenliang. "Doing Topology Optimization Explicitly and Geometrically_A New Moving Morphable Components Based Framework" Journal of Applied Mechanics Vol. 81 No. 8 (2014): p. 081009. DOI 10.1115/1.4027609. http://appliedmechanics.asmedigitalcollection.asme.org/article.aspx?doi=10.1115/1.4027609.

[9] Bendsøe, Martin, and Sigmund, Ole. Topology Optimization: Theory, Methods, and Applications. Springer, Berlin, Germany (2003).

[10] Deaton, Joshua, and Grandhi, Ramana. "A Survey of Structural and Multidisciplinary Continuum Topology Optimization: Post 2000" Structural and Multidisciplinary Optimization Vol. 49 No. 1 (2014): pp. 1-38. DOI 10.1007/s00158-013-0956-z. http://link.springer.com/10.1007/s00158-013-0956-z.

[11] Sigmund, Ole, and Maute, Kurt. "Topology Optimization Approaches: A Comparative Review” Structural and Multidisciplinary Optimization Vol. 48 No. 6 (2013): pp. 1031-1055. DOI 10.1007/s00158-013-0978-6. http://link.springer.com/10.1007/s00158-013-0978-6. 
[12] Guest, James, Prévost, Jean, and Belytschko, Ted. "Achieving Minimum Length Scale in Topology Optimization Using Nodal Design Variables and Projection Functions" International Journal for Numerical Methods in Engineering Vol. 61 No. 2 (2004): pp. 238-254. DOI 10.1002/nme.1064. http://doi.wiley.com/10.1002/nme.1064.

[13] Deng, JiaDong, and Chen, Wei. "Design for Structural Flexibility Using Connected Morphable Components Based Topology Optimization" Science China Technological Sciences Vol. 59 No. 6 (2016): pp. 839-851. DOI 10.1007/s11431-016-6027-0. http://link.springer.com/10.1007/s11431-016-6027-0.

[14] Norato, Julian, Bell, B.K., and Tortorelli, Daniel. “A Geometry Projection Method for Continuum-Based Topology Optimization with Discrete Elements" Computer Methods in Applied Mechanics and Engineering Vol. 293 (2015): pp. 306-327. DOI 10.1016/j.cma.2015.05.005. http://linkinghub.elsevier.com/retrieve/pii/S0045782515001711.

[15] Zhang, Weisheng, Yuan, Jie, Zhang, Jian, and Guo, Xu. "A New Topology Optimization Approach Based on Moving Morphable Components (MMC) and the Ersatz Material Model" Structural and Multidisciplinary Optimization Vol. 53 No. 6 (2016): pp. 1243-1260. DOI 10.1007/s00158-015-1372-3. http://link.springer.com/10.1007/s00158-015-1372-3.

[16] Bremicker, Michael, Chirehdast, Mehran, Kikuchi, Noboru, and Papalambros, Panos. "Integrated Topology and Shape Optimization in Structural Design" Mechanics of Structures and Machines Vol. 19 No. 4 (1991): pp. 551-587. DOI 10.1080/08905459108905156. http://www.tandfonline.com/doi/abs/10.1080/08905459108905156.

[17] Siddiqi, Kaleem, and Pizer, Stephen, eds. Medial Representations: Mathematics, Algorithms and Applications. Springer, Dordrecht, Netherlands (2008).

[18] Chang, Kuang-Hua, and Tang, Poh-Soong. "Integration of Design and Manufacturing for Structural Shape Optimization” Advances in Engineering Software Vol. 32 No. 7 (2001): pp. 555-567. DOI 10.1016/S0965-9978(00)00103-4. http://linkinghub.elsevier.com/retrieve/pii/S0965997800001034.

[19] Lazarov, Boyan, Wang, Fengwen, and Sigmund, Ole. "Length Scale and Manufacturability in Density-Based Topology Optimization" Archive of Applied Mechanics Vol. 86 No. 1-2 (2016): pp. 189-218. DOI 10.1007/s00419-015-1106-4. http://link.springer.com/10.1007/s00419-0151106-4.

[20] Osanov, Mikhail, and Guest, James. "Topology Optimization for Additive Manufacturing Considering Layer-Based Minimum Feature Sizes." Proceedings of the ASME IDETC/CIE. DETC2017-68383: p. V02AT03A036. Cleveland, OH, August 6, 2017. DOI 10.1115/DETC2017-68383. http://proceedings.asmedigitalcollection.asme.org/proceeding.aspx?doi=10.1115/DETC201768383.

[21] Hoang, Van-Nam, and Jang, Gang-Won. "Topology Optimization Using Moving Morphable Bars for Versatile Thickness Control" Computer Methods in Applied Mechanics and Engineering Vol. 317 (2017): pp. 153-173. DOI 10.1016/j.cma.2016.12.004. http://linkinghub.elsevier.com/retrieve/pii/S0045782516309495.

[22] Driessen, A. M. “Overhang Constraint in Topology Optimisation for Additive Manufacturing: A Density Gradient Based Approach.” PhD Thesis. Delft University of 
Technology, Delft, Netherlands. 2016. http://resolver.tudelft.n1/uuid:f95235fc-4e81-48d2-9e2bba03d3c734d5.

[23] Gaynor, Andrew. "Topology Optimization Algorithms for Additive Manufacturing." $\mathrm{PhD}$ Thesis. John Hopkins University, Baltimore, MD. 2015. https://jscholarship.library.jhu.edu/handle/1774.2/38009.

[24] Guo, Xu, Zhou, Jianhua, Zhang, Weisheng, Du, Zongliang, Liu, Chang, and Liu, Ying. "Self-Supporting Structure Design in Additive Manufacturing through Explicit Topology Optimization" Computer Methods in Applied Mechanics and Engineering Vol. 323 (2017): pp. 27-63. DOI 10.1016/j.cma.2017.05.003. http://linkinghub.elsevier.com/retrieve/pii/S004578251631564X.

[25] Langelaar, Matthijs. "An Additive Manufacturing Filter for Topology Optimization of PrintReady Designs" Structural and Multidisciplinary Optimization Vol. 55 No. 3 (2017): pp. 871883. DOI 10.1007/s00158-016-1522-2. http://link.springer.com/10.1007/s00158-016-1522-2.

[26] Svanberg, Krister. "The Method of Moving Asymptotes - a New Method for Structural Optimization" International Journal for Numerical Methods in Engineering Vol. 24 No. 2 (1987): pp. 359-373. DOI 10.1002/nme.1620240207. http://doi.wiley.com/10.1002/nme.1620240207.

[27] Andreassen, Erik, Clausen, Anders, Schevenels, Mattias, Lazarov, Boyan, and Sigmund, Ole. "Efficient Topology Optimization in MATLAB Using 88 Lines of Code" Structural and Multidisciplinary Optimization Vol. 43 No. 1 (2011): pp. 1-16. DOI 10.1007/s00158-010-05947. http://link.springer.com/10.1007/s00158-010-0594-7. 